\title{
The association of asthma, nasal allergies, and positive skin prick tests with obesity, leptin, and adiponectin
}

\begin{abstract}
R. B. Newson ${ }^{1}$, M. Jones ${ }^{2}$, B. Forsberg ${ }^{3}$, C. Janson ${ }^{4}$, A. Bossios ${ }^{5}$, S.-E. Dahlen ${ }^{6}$, E. M. Toskala ${ }^{78}$, A. Al-Kalemji ${ }^{9}$, M. L. Kowalski ${ }^{10}$, B. Rymarczyk ${ }^{11}$, E. M. Salagean ${ }^{12}$, C. M. van Drunen ${ }^{13}$, C. Bachert ${ }^{14,15}$, T. Wehrend ${ }^{16}$, U. Krämer ${ }^{17}$, A. Mota-Pinto ${ }^{18}$, P. Burney ${ }^{1}$, B. Leynaert ${ }^{19,20}$ and D. Jarvis ${ }^{1}$

${ }^{1}$ Respiratory Epidemiology and Public Health Group, Imperial College London, National Heart and Lung Institute, London, UK, ${ }^{2}$ Occupational and Environmental Medicine, Imperial College London, National Heart and Lung Institute, London, UK, ${ }^{3}$ Occupational and Environmental Medicine, Umeå University, Umeå, Sweden, ${ }^{4}$ Department of Medical Sciences: Respiratory Medicine and Allergology, University of Uppsala, Uppsala, Sweden, ${ }^{5}$ Krefting Research Centre, Institute of Medicine, University of Gothenburg, Gothenburg, Sweden, ${ }^{6} \mathrm{CfA}$ - The Centre for Allergy Research, Karolinska Institute, Stockholm, Sweden, ${ }^{7}$ Skin and Allergy Hospital, Helsinki University, Helsinki, Finland, ${ }^{8}$ Department of ORL-HNS, Temple University, Philadelphia, PA, USA, ${ }^{9}$ Odense University Hospital, Odense University, Odense, Denmark, ${ }^{10}$ Department of Immunology, Rheumatology and Allergy, Medical University of Lodz, Lodz, Poland, "'Department of Allergology and Clinical Immunology, Medical University of Silesia, Katowice, Poland, ${ }^{12}$ Department of Allergy and Immunology, Southampton General Hospital, Southampton, UK, ${ }^{13}$ Department of Otorhinolaryngology, Academic Medical Center, Amsterdam, The Netherlands, ${ }^{14}$ University of Ghent, Ghent, Belgium, ${ }^{15}$ Division of Ear, Nose, and Throat Diseases, Karolinska Institute, CLINTEC, Stockholm, Sweden, ${ }^{16}$ Department of Pediatric Pneumonology and Immunology, Charité-Universitätsmedizin Berlin, Berlin, Germany, ${ }^{17}$ IUF - Leibniz Research Institute for Environmental Medicine, Düsseldorf, Germany, ${ }^{18}$ Faculty of Medicine, University of Coimbra, Coimbra, Portugal, ${ }^{19}$ Unit 700 - Epidemiology, INSERM, Paris, France and ${ }^{20}$ Université Paris-Diderot Paris 7, Paris, France
\end{abstract}

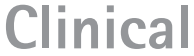 Ct
Experimental
Allergy}

Correspondence:

Roger B. Newson, Respiratory

Epidemiology and Public Health Group, National Heart and Lung Institute, Imperial College London, Royal Brompton Campus, Room 33, Emmanuel Kaye Building, 1B Manresa Road, London SW3 6LR, UK.

E-mail: r.newson@imperial.ac.uk Cite this as: R. B. Newson, M. Jones, B. Forsberg, C. Janson, A. Bossios, S.E. Dahlen, E. M. Toskala, A. Al-Kalemji, M. L. Kowalski, B. Rymarczyk, E. M. Salagean, C. M. van Drunen, C. Bachert, T. Wehrend, U. Krämer, A. Mota-Pinto, P. Burney, B. Leynaert and D. Jarvis, Clinical \& Experimental Allergy, 2014 (44) 250-260.

\section{Summary}

Background Cross-sectional and longitudinal reports show that obese adults have more asthma than non-obese adults. A proposed mechanism is via effects of adipokines (leptin and adiponectin) on the immune system.

Objective We wished to measure the associations of asthma and other atopic diseases with serum adipokine levels and to find whether the associations with asthma were strong enough to rule out the possibility that they are secondary to the association of fatness measures with asthma.

Methods The Global Asthma and Allergy Network of Excellence (GA2LEN) clinical followup survey is a clinical survey, embedded in a larger multi-centre cross-sectional postal survey, involving, with a case/control design, enrichment of the sample with subjects with asthma and chronic rhinosinusitis (CRS). We recorded serum leptin or adiponectin in 845 men and 1110 women in 15 centres and also anthropometric measures of fatness including body mass index and waist/hip ratio, current asthma, and specific skin prick and IgE sensitisation. We used inverse sampling-probability-weighted rank and regression statistics to measure population associations of disease outcomes with adipokines in males and females, adjusting for confounders (area, age, smoking history, and number of elder siblings) and also mutually adjusting associations with adipokines and fatness measures. Results One thousand nine hundred and fifty-five subjects aged 16-77 years had information on leptin or adiponectin levels. Leptin and leptin/adiponectin ratio were positively associated with the level of asthma, especially in females (Somers' $D$ of leptin by asthma score, 0.20 ; 95\% CI, 0.08-0.30; $P=0.00079$ ). These associations were attenuated after adjusting for confounders and became non-significant after additionally adjusting for fatness measures and multiple comparisons.

Conclusions and Clinical Relevance Asthma levels are positively associated with serum leptin. However, we cannot rule out the possibility that this association is secondary to associations of both with fatness measures.

Keywords adiponectin, asthma, IgE sensitivity, leptin, nasal allergy, obesity, skin prick sensitivity

Submitted 20 May 2013; revised 07 August 2013; accepted 14 August 2013 


\section{Introduction}

Cross-sectional and longitudinal reports show that obese adults have more asthma than their non-obese contemporaries [1], but the relationship of atopy with obesity is less clear [2-4]. Several mechanisms have been proposed to explain the relationship of asthma symptoms with obesity, including direct mechanical effects, gastro-oesophageal reflux, perturbation of sex hormone levels, abnormal insulin handling, common genetic predisposition [5, 6], and the role of adipocytederived hormones (in particular leptin and adiponectin) [7].

Leptin is a pro-inflammatory cytokine, structurally related to interleukin-6 (IL-6), that controls food intake and energy expenditure and is raised in those who are obese [8]. The overall effect on $\mathrm{T}$ memory cells is to increase Th- 1 responses and decrease Th-2 responses [7]. Adiponectin, which is lower in those who are obese, is considered to have an overall anti-inflammatory action, possibly by counteracting the production and effects of cytokines such as TNF- $\alpha$, IL- 6 and nuclear factor $-\mathrm{kB}[7]$.

To date, large-scale epidemiological studies that have examined the relationship of asthma with adipokines in adults [9-12] show inconsistent relationships that, in some studies, appear gender specific $[9,11]$. In the Dunedin cohort [11], asthma symptoms were not associated with leptin levels in men or in women, but men (not women) with higher levels of adiponectin had lower exhaled nitric oxide levels. Measures of fatness are highly correlated with adipokine levels, and researchers have used different approaches to untangle their relative effects, including mutual adjustment, and examination of the effects of adipokine in thin and then in fat individuals. No clear picture has emerged to date, and to our knowledge, only one population-based epidemiological study has reported these relationships with allergic sensitisation as measured by skin prick tests. That study suggested increased risk of sensitisation to any common environmental aeroallergen with higher leptin levels in young adults [13] in Germany.

The aim of this report is to describe the cross-sectional association of obesity and adipokines with asthma in a large sample of men and women living in Europe who took part in the Global Asthma and Allergy Network of Excellence (GA $\left.{ }^{2} \mathrm{LEN}\right)$ epidemiological survey.

\section{Materials and methods}

\section{Study design, setting, and participants}

A representative sample of adults aged 20-75 years, living in 25 centres in Europe, took part in a postal survey asking about symptoms of asthma and chronic rhinosinusitis (CRS). Eighteen centres in 11 countries invited a sample of responders to the postal survey for a nested clinical follow-up. Invitation to follow-up was dependent on symptoms reported at the postal survey and aimed to recruit representative samples of participants in four postal survey disease groups: (1) asthma and CRS, (2) asthma (but no CRS), (3) CRS (but no asthma), and (4) controls (neither asthma nor CRS). The postal survey was carried out from October 2007 to December 2008, and the clinical surveys from September 2008 to January 2010. The methods, response, and results to the postal survey have been reported [14, 15].

\section{Information collected}

After completion of an interviewer-administered questionnaire, height, weight, waist circumference, and hip circumference were recorded, and body mass index (BMI), waist/hip ratio, and waist/height ratio were calculated. Skin prick tests were taken to a panel of indoor allergens (Dermatophagoides pteronyssinus, Dermatophagoides farinae and Blattella), pets (cat, dog), pollens (grass mix, Timothy grass, Artemisia, Parietaria, olive. birch), and mould (Alternaria alternata). Non-fasting blood samples were taken and serum was measured for serum-specific IgE to grass (ImmunoCAP; Thermo Fisher, Uppsala, Sweden) in a single laboratory [Rijksinstituut voor Volksgezondheid en Milieu (RIVM), Amsterdam, Netherlands]. IgE sensitisation to Timothy grass was considered present when serum measures were $>0.35 \mathrm{kU} / \mathrm{L}$, and SPTs were positive if the mean wheal diameter was $>0 \mathrm{~mm}$ [16]. Participants with a positive negative control (saline) or a negative positive control (histamine dihydrochloride $10 \mathrm{mg} / \mathrm{mL}$ ) were excluded. From the participants with serum samples in each case status group in each centre, we measured adipokines on the full number of participants or on a subsample of 44 (whichever was less), because of cost constraints. Serum leptin and adiponectin were measured using a MULTI-ARRAY ${ }^{\circledR}$ assay system (Meso Scale Discovery, Gaithersburg, MD, USA) in all available samples from participants with asthma and participants with CRS, and a random sample of participants with neither condition in a single laboratory (Imperial College, London). Sera were used neat for leptin and in $1: 1000$ dilution for the adiponectin assay. The lower limits of detection were $0.001 \mathrm{pg} / \mathrm{mL}$ for leptin and $85 \mathrm{pg} / \mathrm{mL}$ for adiponectin, and measurements below these were recoded to the detectability limits. Three centres (Krakow, Poland; Skopje, Macedonia; Palermo, Italy) had insufficient serum samples for adipokine measures.

Asthma was defined as reporting ever having asthma and at least one of the following symptoms in the last 
12 months: (1) wheeze or whistling in the chest or (2) waking with chest tightness or (3) waking with shortness of breath or (4) waking with an attack of coughing. The European Community Respiratory Health Survey (ECRHS) asthma score (on a scale from 0 to 5) was derived by summing responses to questions on (1) wheeze with breathlessness, (2) chest tightness, (3) attack of shortness of breath (SOB) coming on at rest, (4) SOB after exercise, and (5) being woken by SOB [17].

Allergic rhinitis was defined as a positive response to the question: 'Do you have any nasal allergies including hayfever?'

\section{Statistical methods}

Full statistical methods are detailed in the online supporting information.

The association of asthma, nasal allergies, serum IgE to Timothy grass, positive skin prick tests (SPT) to house dust mite (positive SPT to either of Dermatophagoides pteronyssinus or Dermatophagoides farinae), positive SPT to Timothy grass, and positive SPT to any of the allergens tested with three adipokine measures [leptin, adiponectin, leptin/adiponectin ratio (LAR)] and four anthropometric measures of fatness (BMI, waist circumference, waist/hip ratio, and waist/height ratio) was examined separately in men and in women using Somers' $D[18,19]$. Somers' $D$ is a rank statistic (lying between -1 and 1) representing the risk of individuals with the outcome of interest (e.g. asthma, nasal allergies) having a higher predictor level (e.g. leptin, BMI) compared with those without the outcome. It has been used here because it is suitable for both binary outcomes and for the asthma score, it can be determined within strata specified by confounders, and it is resistant to the extreme effects of outliers. A Somers' $D$ of 0.1 in the relationship of asthma with leptin implies that it is 10\% more likely that asthmatics have higher leptin levels than non-asthmatics than the other way round (that is, compared with the likelihood that nonasthmatics have higher leptin levels than asthmatics).

We used two propensity scores to adjust the analysis [20]. The first propensity score adjusted for area, age, smoking status, pack years of smoking, and number of elder siblings, and the second propensity score, in addition, adjusted for fatness measures (when examining associations with adipokine levels) or adipokine levels (when examining associations with fatness measures).

To allow for multiple comparisons, we applied the Simes-Benjamini-Hochberg procedure [21] to generate $Q-$ values [19] for the $98 P$-values for Somers' $D$ for trend (seven outcomes $\times$ seven exposures $\times$ two genders) for each of the three adjustments [unadjusted, propensity score (1) adjusted and propensity score (2) adjusted].
These $Q$-values are equivalent to the lowest false discovery rate (FDR) that could be set to reject the null hypothesis and are also known as 'adjusted $P$-values'.

As participants in the clinical survey were initially recruited from postal survey responders using case-control selection procedures, analyses were weighted using sampling-probability weights. The associations presented are estimates of those that we would expect to find in postal survey responders, had they all been assayed for adipokines in the clinical survey. In Poland, Sweden and UK, there were small numbers of subjects in some case groups, and to avoid some individuals having extreme weights, subjects from centres have been combined to form multi-centre areas (Sweden, UK and Poland).

\section{Results}

Figure 1 details the selection of the sample for these analyses. Three thousand five hundred and five subjects from 18 centres contributed data to the GA ${ }^{2} L E N$ follow-up survey. Of these, 55 had unknown gender or questionnaire age either unknown or below 16 years, and a further 192 were in three centres (Palermo, Krakow and Skopje) which were not included in measurement of either leptin or adiponectin. One thousand three hundred and three subjects in the remaining 15 centres had no measurements of either leptin or adiponectin, because the adipokine study was a nested-nested study inside the GA ${ }^{2}$ LEN follow-up survey, which in turn was nested in the GA ${ }^{2} L E N$ postal survey. Leptin or adiponectin levels were therefore available on a subsample of 1955 subjects of known gender (845 men and 1110 women) aged 16 years or over in 15 centres in nine countries. Further descriptive data on this sample are given in Tables 1 and 2. Note that, in some centres in some case groups, there were $<44$ participants per case status group per centre who also had serum samples.

Figure 2 (duplicated in online Figure S2) shows the confounder-adjusted associations. (See online Figures S1 and S3, for unadjusted and mutually adjusted associations, respectively.) In women (but not men), asthma was positively associated with measure of fatness (BMI $P=0.38$, waist circumference $P=0.079$, waist/hip ratio $P=0.052$, waist/height ratio $P=0.031$ ). In both men and women, higher asthma score was positively associated with fatness measures (in women, BMI $P=0.025$, waist circumference $P=0.028$, waist/hip ratio $P=0.049$, waist/height ratio $P=0.0065$; in men, BMI $P=0.085$, waist circumference $P=0.38$, waist/hip ratio $P=0.26$, waist/height ratio $P=0.095)$. In both men and women, there was evidence that higher asthma score was associated with higher leptin and leptin/ adiponectin ratio (in women, leptin $P=0.0055$, 


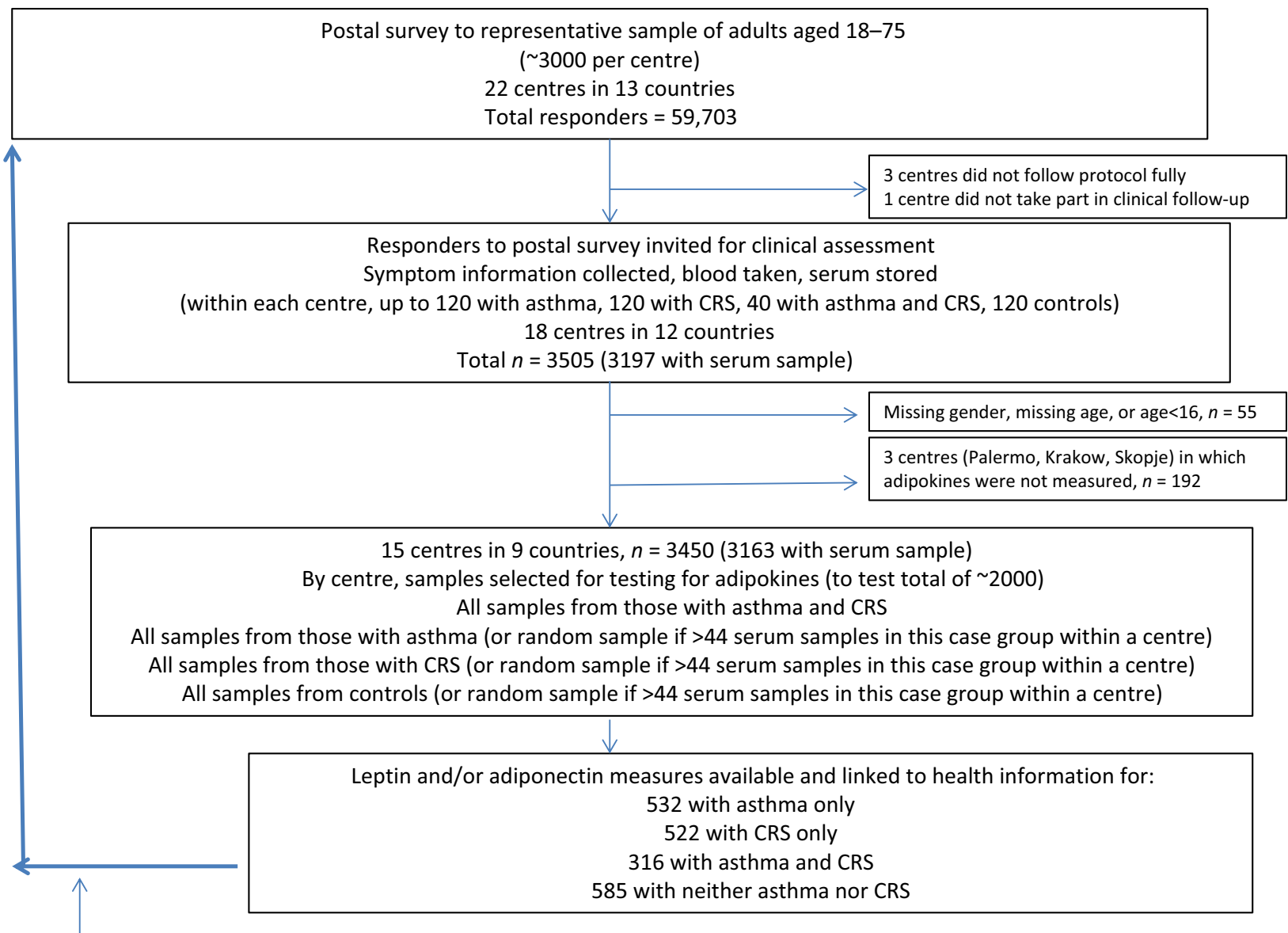

Reweighting procedure so that results represent associations that would have existed in the 15 centres (9 countries), had all those who responded to postal survey had clinical measures and serum leptin and adiponectin measured

Fig. 1. Selection of the sample for analysis from the subjects in the GA ${ }^{2}$ LEN clinical survey. GA ${ }^{2}$ LEN, Global Asthma and Allergy Network of Excellence.

adiponectin $P=0.27$, leptin/adiponectin ratio $P=0.03$; in men, leptin $P=0.082$, adiponectin $P=0.85$, leptin/ adiponectin ratio $P=0.3$ ). However, allowing for multiple comparisons, none of these associations are present at adjusted $P<0.2$. No clear associations were observed for nasal allergies, IgE sensitisation to Timothy grass, or positive skin prick tests.

The full set of Somers' $D$ statistics for overall trend is given in the online repository (online Figures S1-S3). Figure S1 confirms that the ECRHS 5-point asthma score possibly has positive unadjusted associations with leptin and leptin/adiponectin ratio, at least in females, even after allowing for multiple comparisons (adjusted $P<0.10$ ). However, Figure S3 confirms that, after mutual adjustment and correction for multiple comparisons, all adjusted $P$-values are non-significant.

Table 3 details the change in association between the ECRHS 5-point asthma score and the three adipokine and four fatness measures, when we adjusted first for age, sex and smoking history ['propensity-adjusted (1)']
Table 1. Number of participants in the clinical study and with cytokine measures by case status in each study area

\begin{tabular}{|c|c|c|c|c|c|}
\hline Study area & Control & Asthma & CRS & $\begin{array}{l}\text { Asthma } \\
\text { \& CRS }\end{array}$ & Total \\
\hline $\begin{array}{l}\text { Sweden (Umea, } \\
\text { Uppsala, Gothenburg, } \\
\text { Stockholm) }\end{array}$ & 147 & 176 & 154 & 139 & 616 \\
\hline Helsinki & 46 & 39 & 28 & 12 & 125 \\
\hline Odense & 42 & 45 & 44 & 39 & 170 \\
\hline $\begin{array}{l}\text { Poland (Lodz, } \\
\text { Katowice*) }\end{array}$ & 61 & 30 & 57 & 16 & 164 \\
\hline $\begin{array}{l}\text { UK (London, } \\
\text { Southampton) }\end{array}$ & 50 & 59 & 34 & 16 & 159 \\
\hline Amsterdam & 43 & 43 & 44 & 10 & 140 \\
\hline Ghent & 64 & 21 & 46 & 16 & 147 \\
\hline Brandenburg & 44 & 43 & 44 & 14 & 145 \\
\hline Duisburg & 44 & 32 & 27 & 17 & 120 \\
\hline Coimbra & 44 & 44 & 44 & 37 & 169 \\
\hline Total & 585 & 532 & 522 & 316 & 1955 \\
\hline
\end{tabular}

*No subjects in Krakow had data on leptin or adiponectin. CRS, chronic rhinosinusitis. 
Table 2. Information at follow-up on participants included in the analysis*

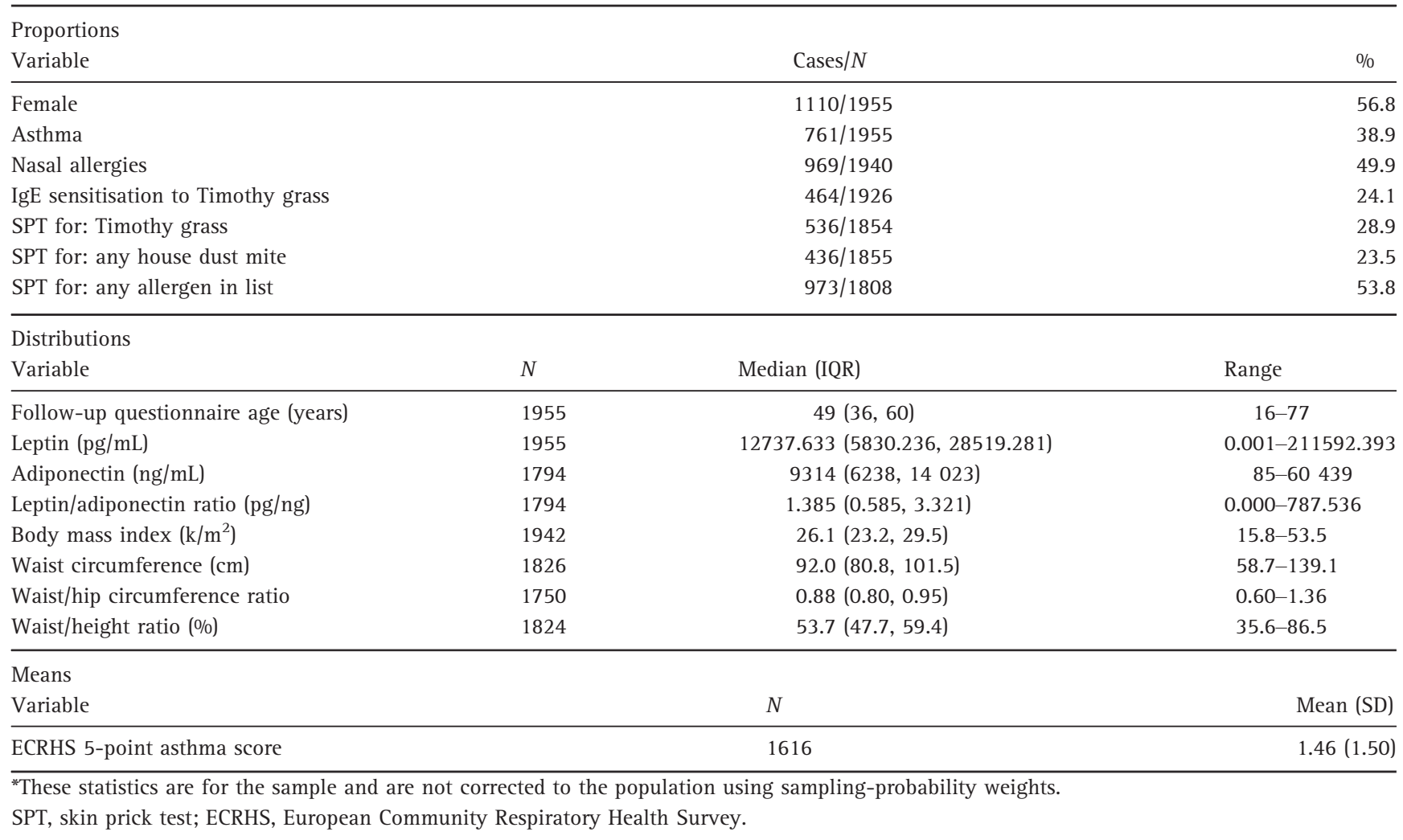

and additionally for fatness measures (in the case of adipokines) and for adipokines (in the case of fatness measures) ['propensity-adjusted (2)']. We see that adjustment attenuates the trend and widens the confidence intervals, with the result that we cannot disentangle the independent effects of adipokines (on the one hand) and fatness measures (on the other), although both fatness measures and adipokines predict asthma score. This attenuation through mutual adjustment is not surprising, in view of the high level of correlation, within study areas and genders, between adipokine measures and fatness measures. (See online Table S1, which gives the within-area Somers' $D$ statistics of each fatness measure with respect to each adipokine measure, showing that, in both males and females, fatness measures are strongly positively associated with leptin and leptin/adiponectin ratio and less strongly negatively associated with adiponectin.)

In a post hoc analysis, we examined the association of ECRHS asthma score with adipokine levels within each BMI group (Fig. 3). The number of participants with $\mathrm{BMI}<20$ was small. There was some suggestion that an association of ECRHS asthma score with the leptin/adiponectin ratio was present even in women who were underweight $(P=0.001)$ or normal weight $(P=0.13)$. However, confidence intervals are wide, and we cannot exclude that these observations arise from multiple subgroup analyses.
In another post hoc analysis, we measured the within-area associations between asthma (and asthma score) and the adipokine and fatness measures, in atopics and non-atopics (atopy was defined as positive SPT to at least one of the tested allergens). This was carried out using the unadjusted (see online Figures S4 and S5) and propensity-adjusted Somers' $D$ statistics of predictors (associations with asthma score shown in Fig. 4). We see little evidence of differences in these associations in atopics and non-atopics, although the confidence intervals are wider, because of the smaller sample sizes.

\section{Discussion}

In this large multi-centre cross-sectional study, we provide further evidence that obesity and leptin are associated with asthma, but not allergy, in adults. Leptin and obesity measures are correlated, and we could not demonstrate independent associations of either with asthma. Our post hoc observation that the higher leptin/adiponectin ratio is associated with more symptoms in women with low and normal BMI may suggest independent associations with adipokines, but this may have arisen by chance, due to the large number of statistical tests that we have conducted.

Previous epidemiological studies in adults examining the relationship of asthma with leptin have produced 


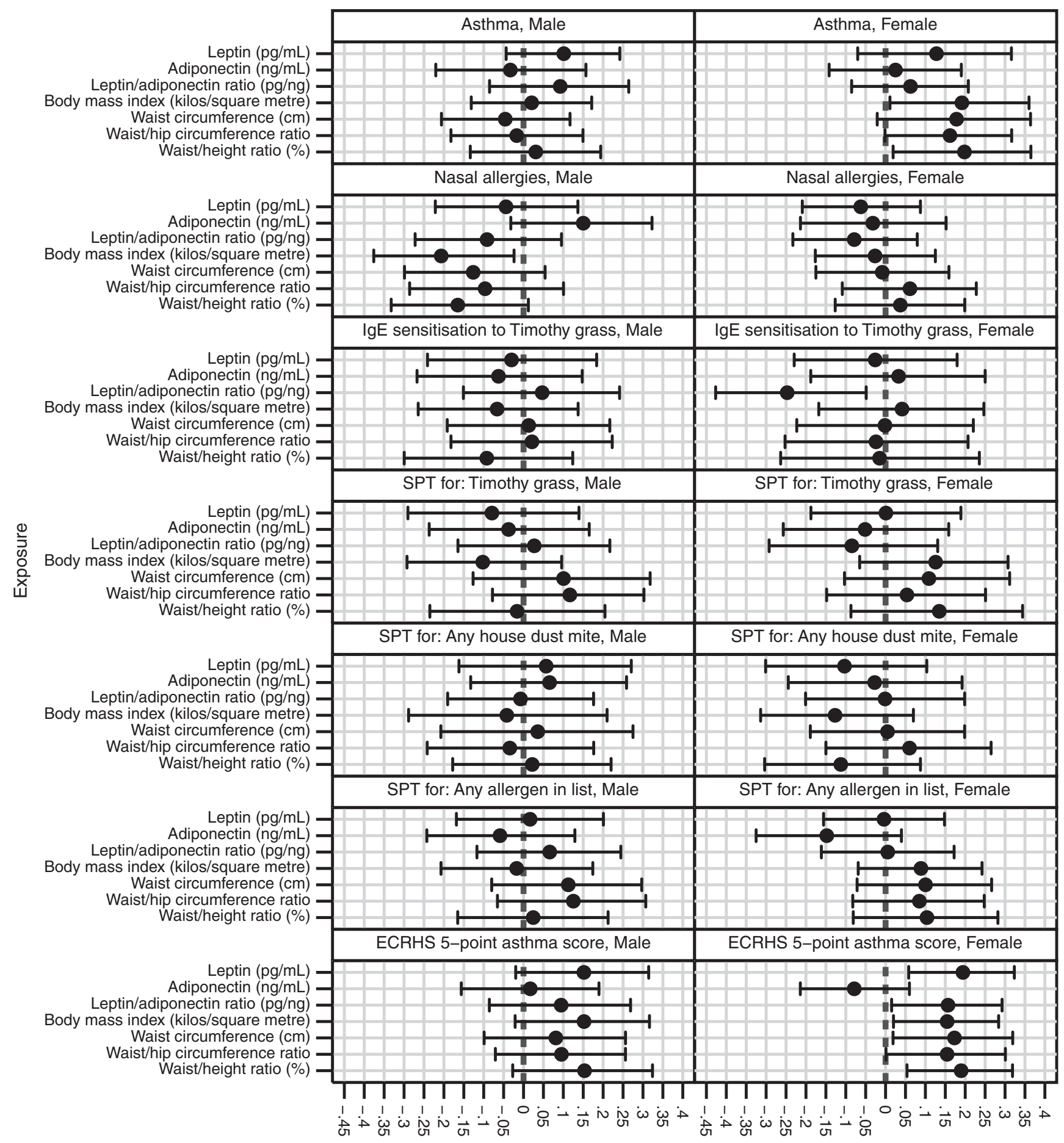

Somers' $D$ (Propensity-adjusted (1), with $95 \% \mathrm{Cl}$ ) of exposure with respect to outcome

Graphs by Outcome and Gender

Fig. 2. Somers' $D$ of associations of fatness measures and adipokine measures with asthma, nasal allergies, and atopy with adjustment for confounders only (propensity score 1).

inconsistent results. Using information from adults surveyed as part of the National Health and Nutrition Examination Survey in USA, Sood et al. [9] reported strong associations of physician-diagnosed asthma with leptin in women (but not men), with the associations being much stronger in those who were under the age of 50 (that is, assumed to be premenopausal). Sood et al. also observed that the strength of these associa- 
Table 3. Unadjusted, confounder-adjusted and mutually adjusted means for ECRHS 5-point asthma score in women by adipokine tertiles and fatness groups, with Somers' $D$ for trend

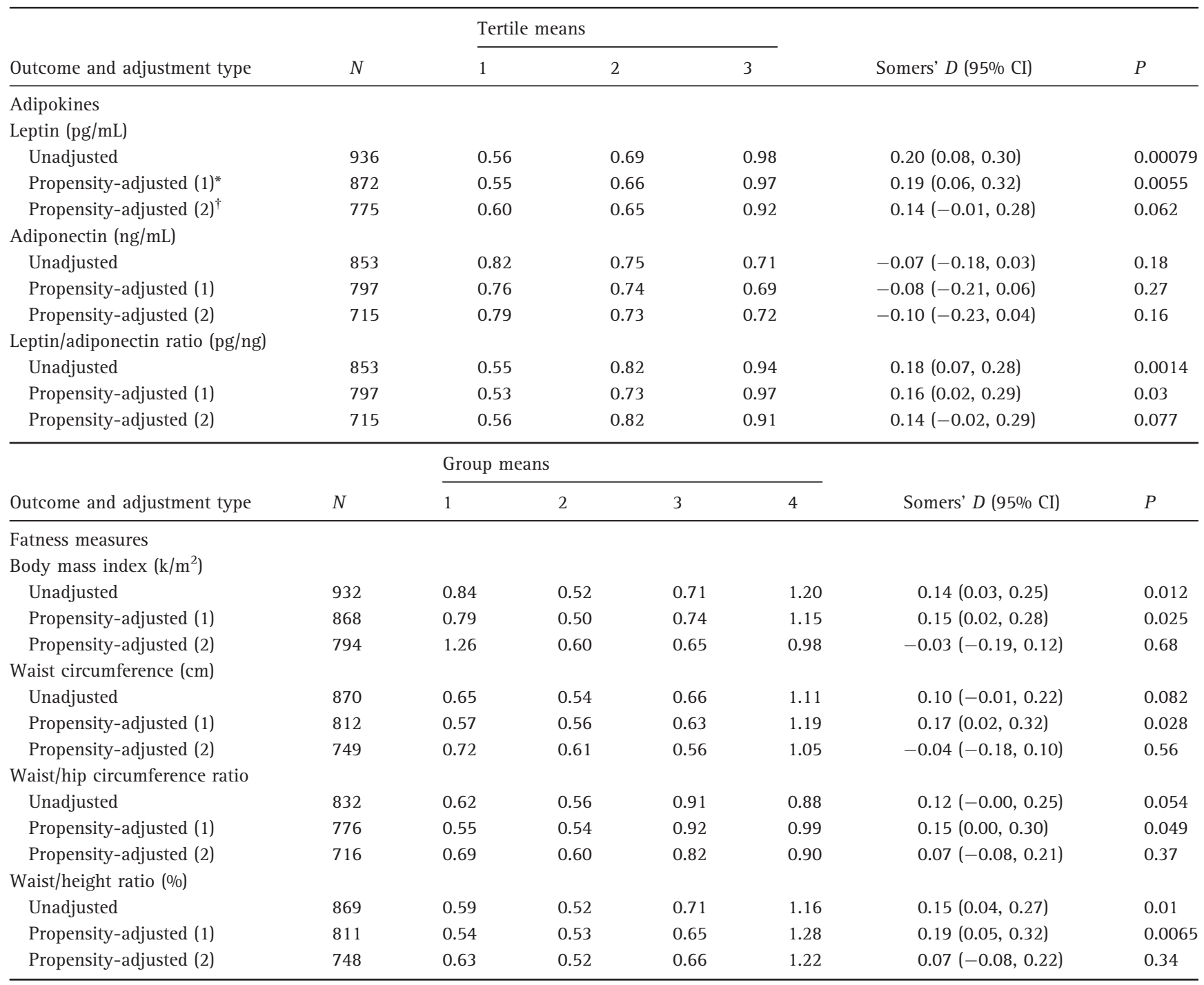

*Adjusted for age and smoking history.

${ }^{\dagger}$ Adjusted for age and smoking history and for fatness measures (in the case of adipokine exposures) and for adipokine exposures (in the case of fatness measures).

Tertile cut-offs for adipokines in men: leptin (pg/mL) < 4556, 4556-10 191, 10 192+: adiponectin (ng/mL) < 6321, 6321-9926, 9927+: LAR (pg/ ng) $<0.5599$, 0.5599-1.455, 1.456+.

Tertile cut-offs for adipokines in women: Leptin $(\mathrm{pg} / \mathrm{mL})<13$ 704, 13 704-32 158, 32 159+: Adiponectin (ng/mL) < 8305, 8305-14 241, 14 242+: LAR $(\mathrm{pg} / \mathrm{ng})<1.19,1.19-3.359,3.360+$.

Group cut-offs for BMI: $[0,20),[20,25),[25,30)$ and $30+\mathrm{k} / \mathrm{m}^{2}$.

ECRHS, European Community Respiratory Health Survey.

tions varied depending on what adjustment for 'fatness' was included in the analysis. Correction for triceps skinfold thickness made little difference, but inclusion of a term for BMI abolished the apparent association. Sutherland et al. [11] found little evidence that leptin (or adiponectin) levels were associated with asthma, wheeze, and lung function in 32-year-old men or women taking part in the Dunedin cohort study. Adjustment for percentage body fat made little difference to the overall conclusion. In the Cardiovascular Risk in Young Finns Study, Jartti et al. [12] showed an association of asthma with leptin in adults aged 24-39 years, but this association was not present after adjustment for several factors including BMI. They concluded that leptin played little role in asthma.

We found no conclusive cross-sectional association of any of the health outcomes with adiponectin level. The CARDIA study [10] showed similar adiponectin 


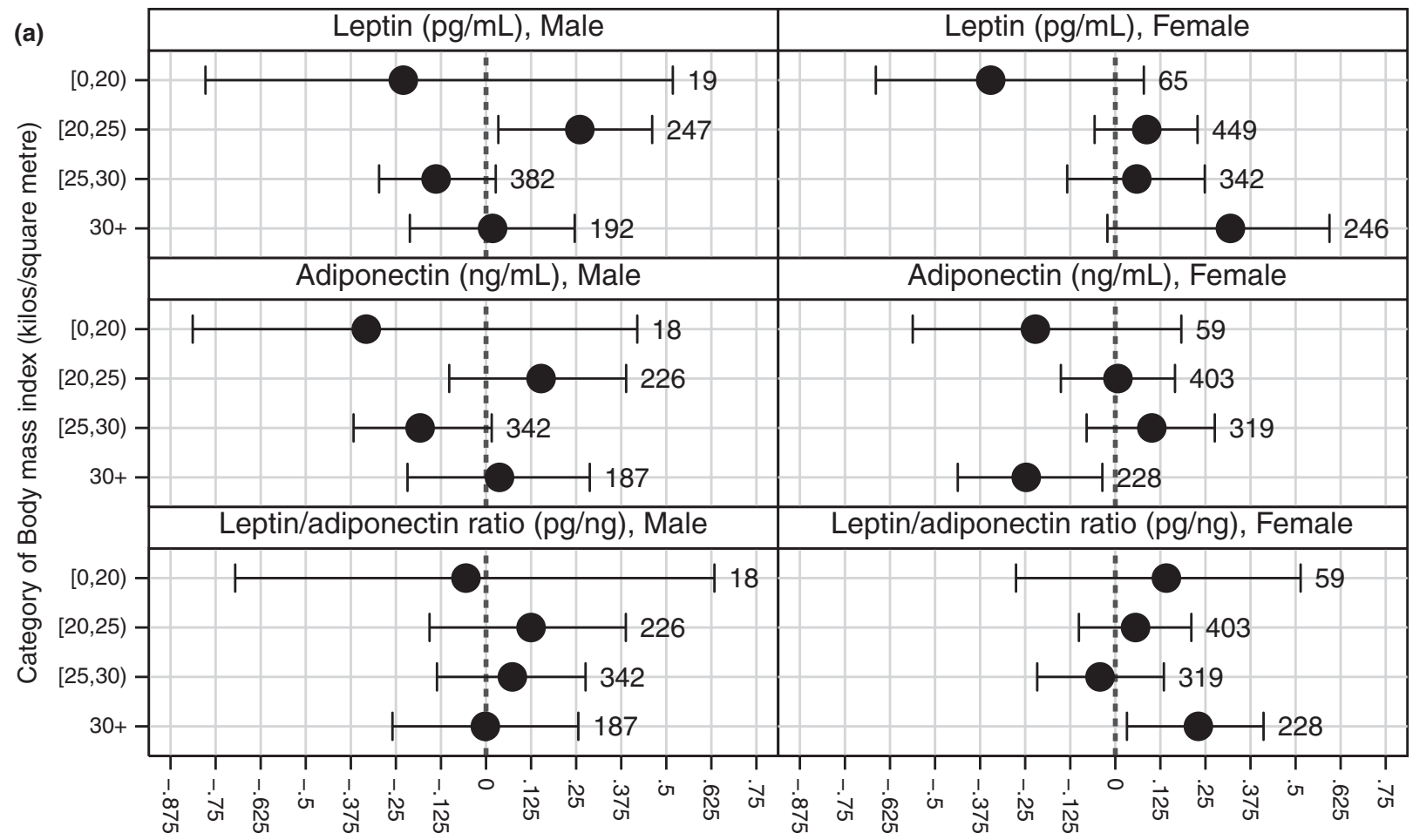

Graphs by Exposure and Gender

Somers' $D(95 \% \mathrm{Cl}, \mathrm{N})$ of cytokine exposures with respect to: Asthma

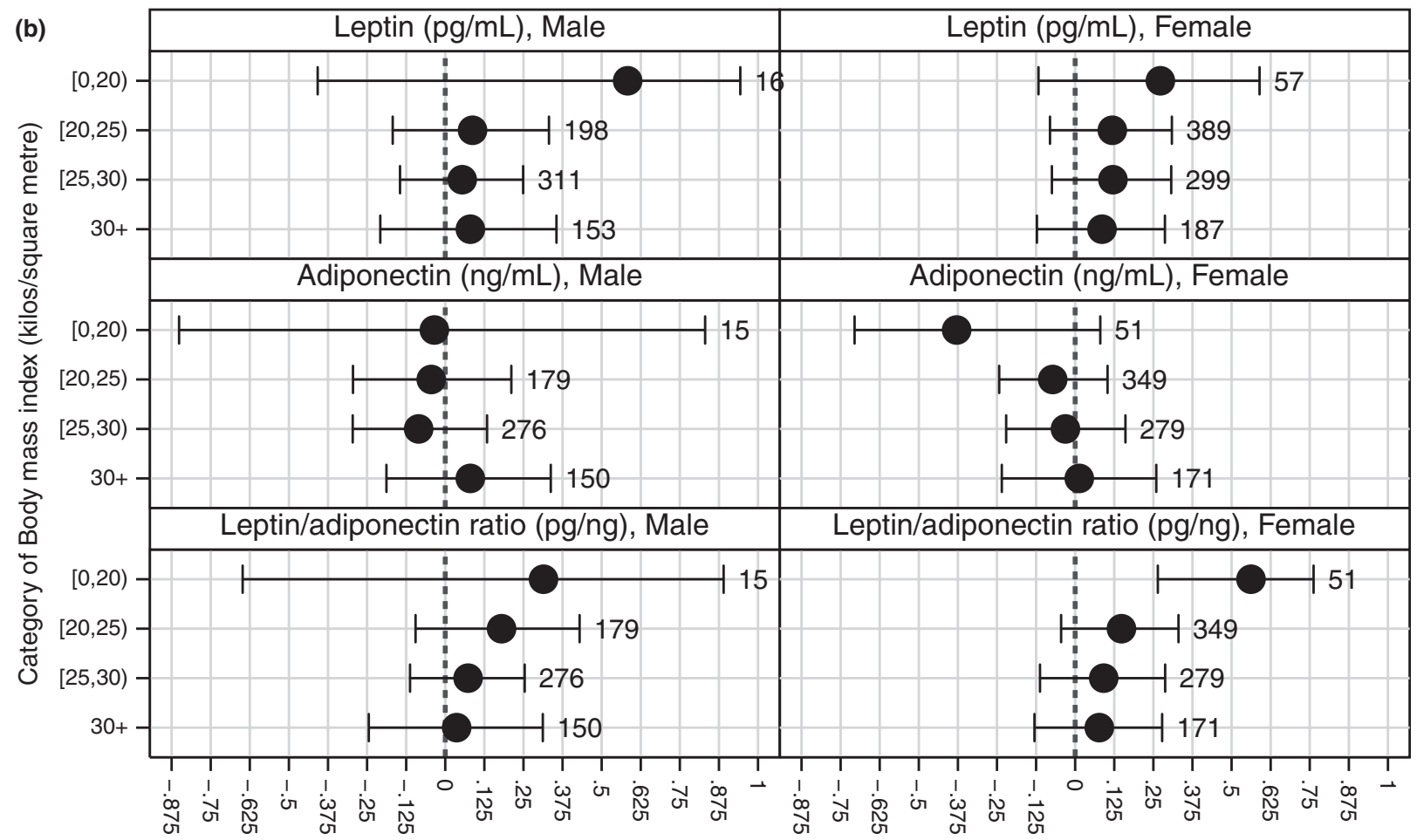

Somers' $D(95 \% \mathrm{Cl}, \mathrm{N})$ of cytokine exposures with respect to: ECRHS 5-point asthma score

Graphs by Exposure and Gender

Fig. 3. Unadjusted Somers' $D$ of associations of fatness measures and adipokine measures with (a) asthma and (b) ECRHS asthma score stratified by BMI. ECRHS, European Community Respiratory Health Survey; BMI, body mass index. 


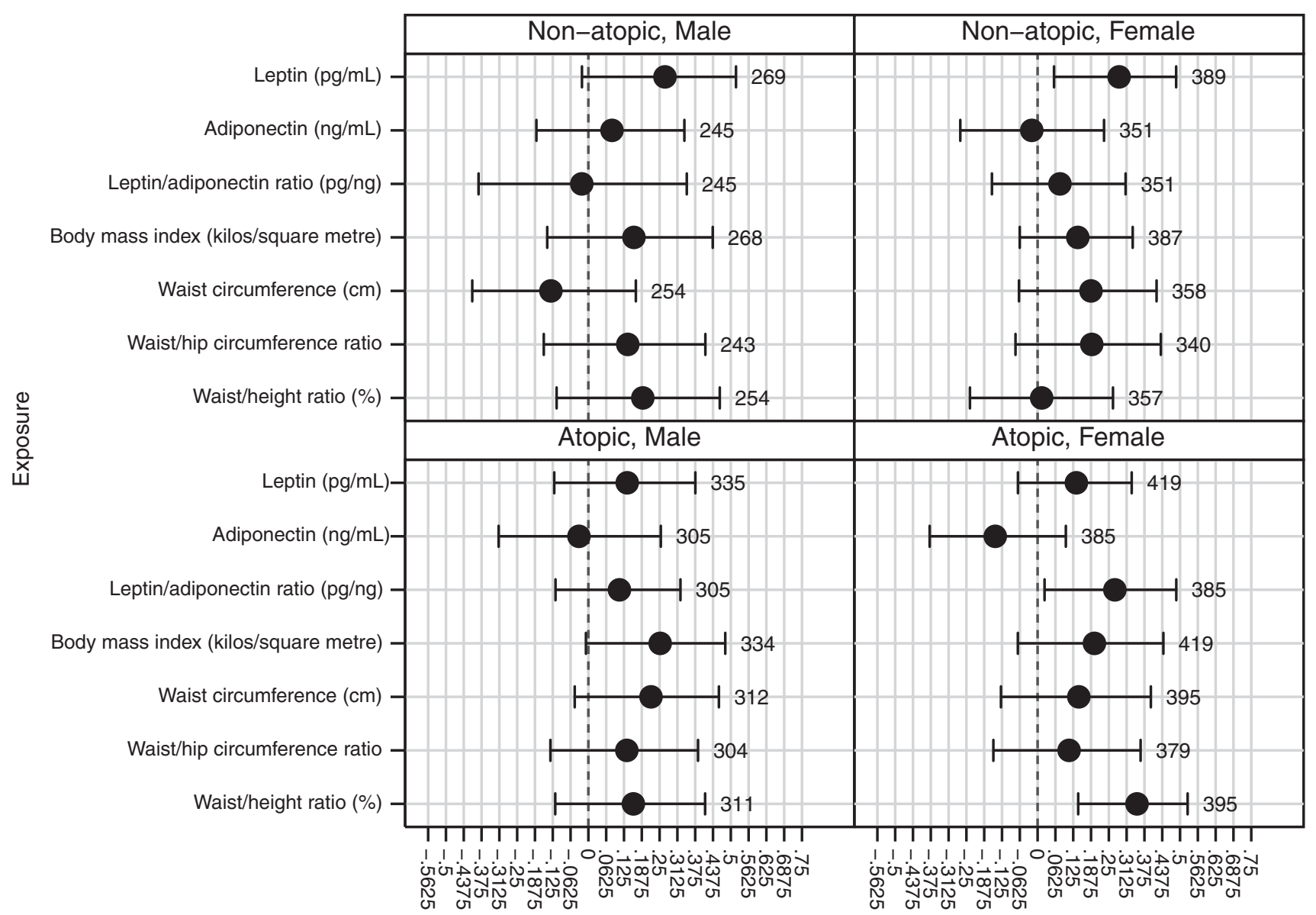

Somesr' $D$ (Propensity-adjusted (1), with $95 \% \mathrm{Cl}$ and $\mathrm{N}$ ) of exposure with respect to: ECRHS 5-point asthma score Graphs by Skin prick atopy to any allergen in list and Gender

Fig. 4. Associations of adipokine and fatness measures with respect to ECRHS 5-point asthma score within atopy and gender groups with adjustment for confounders only (propensity score 1). ECRHS, European Community Respiratory Health Survey.

levels in those with and without self-reported asthma, although in subgroup analyses there was a suggestion of a protective role for asthma in pre-menopausal women (even though formal testing to assess interactions by menopausal status did not reach conventional level of statistical significance). We investigated the association of health outcomes with the leptin/adiponectin ratio. This measure is associated with insulin resistance in adults without diabetes [22] and is said to predict the development of diabetes [23], but, like Jartti et al. [12], we found the associations of asthma and allergy with leptin/adiponectin ratio broadly mirrored the associations seen for leptin.

If there are effects of leptin (and other adipokines) on asthma, their high correlation with obesity makes it difficult to assess their independent role. Furthermore, the causal pathway is not clear. Obesity is referred to as a 'leptin resistant' condition, and leptin is produced by adipocytes (therefore high BMI causes high leptin levels). However, leptin is also part of negative feedback loop which suppresses appetite and increases metabolic rate (therefore leptin levels influence BMI). When measures of fatness and adipokines are made at a single time point, it is not entirely clear whether it is appropriate to adjust one for the other, although this is widely carried out in research of metabolic disorders. Epidemiologists examining the associations of breast cancer [24], hypertension [25], and diabetes [26] with obesity and adipokines have also commented on the difficulty in disentangling their effects. This makes any level of causal inference difficult, and we cannot provide any evidence to support the notion that an intervention that would block the immunological effects of adipokines (without blocking their slimming or fattening effects) would influence development of asthma or atopy. Equally, we cannot say (from our data alone) that weight reduction without related changes in adipokine levels would reduce asthma.

Our study is cross-sectional, and as explained in the previous paragraph, we are unable to confirm the 
temporal pattern of the associations observed. Response rates to the postal survey were fair but not high, and we have previously shown that women and older adults were more likely to respond to the survey [14]. There was no evidence that those with symptoms were more likely to respond [14]. Associations observed were similar in centres with high and low response rates, and as only complex response biases can give rise to biased associations in population studies, we believe the associations we report are representative of those seen in European adult populations. We have used sophisticated but appropriate statistical techniques to account for the multi-centre case-control design of the study, to adjust for relevant confounders, and to allow for multiple testing. We have used propensity scores (which offer a statistically efficient approach for facilitating model convergence when some subgroups are small) to adjust for factors of interest. We have presented associations using Somers' $D$ (and its 95\% confidence interval), which allowed a common metric for multiple testing with outcomes that have binary or negative binomial distributions (such as the asthma score). As with all parameters, highly statistically significant estimates may represent small, clinically irrelevant differences, but Somers' $D$ is a good measure for overall ordinal association. We have conducted multiple tests and corrected for this. Ideally, we would have investigated some associations using meta-analyses of each centre's data and including testing of interaction terms for menopausal status in women, but our study, although large, has limited statistical power for such approaches.

In our study, leptin and adiponectin levels were tested on non-fasting samples. As leptin [27] and adiponectin [28] change little following ingestion of food, it is unlikely that our failure to observe strong associations is influenced by random error related to recent ingestion of food.

To our knowledge, no one has reported associations of leptin with rhinitis, and there has only been one epidemiological study of the relationship of allergic sensitisation with adipokines [13] in adults (showing some evidence of a more sensitisation with increasing leptin levels). One further study has reported that leptin was not associated with sensitisation in children [29]. There was no convincing evidence in our data that strong associations exist between obesity/adipokines and these markers of atopy. Higher leptin levels in men were associated non-significantly with a lower prevalence of nasal allergies, but there were no strong associations with sensitisation to Timothy grass. Our study does not support previous work in mice, suggesting that leptin plays a role in the allergic response [30]. If obesity and adipokines are associated with asthma, but not atopy, as suggested by our analysis, we would expect that there will be stronger associations of obesity and adipokines with 'non-atopic asthma' than with 'atopic asthma', as shown and discussed in other publications [31, 32]. In our post hoc analyses, we saw little evidence for such a difference, although this multi-centre study was too small to fully explore these associations.

In summary, we have shown that, in both men and women, asthma is associated with leptin and leptin/ adiponectin ratio, but we were unable to clearly show that this is independent of the relationship of asthma with obesity. This cross-sectional epidemiological study provides little evidence that changes in endogenous leptin or adiponectin levels, without related changes in fatness, would have any benefit for asthma.

\section{Conflict of interest}

This study was conducted as part of the Global Allergy and Asthma European Network (GA $\left.{ }^{2} L E N\right)$ funded through the Sixth European Union Framework programme for research, contract no. F00D-CT- 2004506378.

\section{References}

1 Ford ES. The epidemiology of asthma and obesity. J Allergy Clin Immunol 2005; 115:897-909.

2 Jarvis D, Chinn S, Potts J, Burney P. Association of body mass index with respiratory symptoms and atopy: results from the European Community Respiratory Health Survey. Clin Exp Allergy 2002; 32:831-7.

3 Chen Y, Rennie D, Cormier Y, Dosman J. Association between obesity and atopy in adults. Int Arch Allergy Immunol 2010; 153:372-7.
4 Ma J, Xiao L, Knowles S. Obesity, insulin resistance and the prevalence of atopy and asthma in US adults. Allergy 2011; 65:1456-63.

5 Beuther DA, Weiss ST, Sutherland ER. Obesity and asthma. Am J Respir Crit Care Med 2006; 174:112-9.

6 Sin DD, Sutherland ER. Obesity and the lung: 4 -à obesity and asthma. Thorax 2008; 63:1018-23.

7 Sood A. Obesity, adipokines, and lung disease. J Appl Physiol 2010; 108:744-53.

8 Bastard J, Maachi M, Lagathu C et al. Recent advances in the relationship between obesity, inflammation and insulin resistance. Eur Cytokine Netw 2006; 17:4-6.

9 Sood A, Ford ES, Camargo CA. Association between leptin and asthma in adults. Thorax 2006; 61:300-5.

10 Sood A, Cui X, Qualls C et al. Association between asthma and serum adiponectin concentration in women. Thorax 2008; 63:877-82.

11 Sutherland T, Sears MR, McLachlan CR, Poulton R, Hancox RJ. Leptin, adiponectin and asthma: findings from a population based cohort study. Ann 
Allergy Asthma Immunol 2009; 103:101-7.

12 Jartti T, Saarikoski L, Jartti L et al. Obesity, adipokines and asthma. Allergy 2009; 64:770-7.

13 Radon K, Schulze A, Schierl R, Dietrich-Gumperlein G, Nowak D, Jorres RA. Serum leptin and adiponectin levels and their association with allergic sensitisation. Allergy 2008; 63:144854.

14 Hastan D, Fokkens W, Bachert C et al. Chronic rhinosinusitis in Europe - an underestimated disease. A GA ${ }^{2} \mathrm{LEN}$ study. Allergy 2011; 66:1216-23.

15 Jarvis D, Newson R, Lotvall $\mathrm{J}$ et al. Asthma in adults and its association with chronic rhinosinusitis: the $\mathrm{GA}^{2}$ LEN survey in Europe. Allergy 2012; 67:91-8.

16 Chinn S, Jarvis D, Luczynska CM, Lai E, Burney P. Measuring atopy in a multi-centre epidemiological study. Eur J Epidemiol 1996; 12:155-62.

17 Sunyer J, Pekkanen J, Garcia-Esteban $\mathrm{R}$ et al. Asthma score: predictive ability and risk factors. Allergy 2007; 62:142-8.

18 Harrell FE. Regression modeling strategies with applications to linear models, logistic regression and survival analysis. 1st Edn. New York, NY: Springer, 2001.
19 Newson RB. Frequentist q-values for multiple test procedures. Stata J 2010; 10:568-84.

20 Imai K, van Dyk D. Causal inference with general treatment regimes: generalizing the propensity score. J Am Stat Assoc 2004; 99:854-66.

21 Benjamini Y, Yekutieli D. The control of the false discovery rate in mulitple testing under dependency. Ann Stat 2001; 29:1165-88.

22 Finucane F, Luan J, Wareham N et al. Correlation of leptin:adiponectin ratio with measures of insulin resistance in non-diabetic individuals. Diabetologia 2009; 52:2345-9.

23 Thorand B, Zierer A, Baumert J, Meisinger C, Herder C, Koenig W. Associations between leptin and the leptin/ adiponectin ratio and incident Type 2 diabetes in middle aged men and women:results from the MONICA/ KORA Augsburg Study 1984-2002. Diabet Med 2010; 27:1004-11.

24 Harris HR, Tworoger SS, Hankinson SE, Rosner BA, Michels KB. Plasma leptin levels and risk of breast cancer in premenopausal women. Cancer Prev Res (Phila) 2011; 4:1449-56.

25 Grontved A, Steene-Johannessen J, Kynde I et al. Association between plasma leptin and blood pressure in two population-based samples of chil- dren and adolescents. $J$ Hypertens 2011; 29:1093-100.

26 Schmidt M, Duncan B, Vigo A et al. for the ARIC investigators. Leptin and incident type 2 diabetes; risk or protection? Diabetologia 2006; 49:208696.

27 Korbonitis M, Trainer P, Little $\mathrm{J}$ et al. Leptin levels do not change acutely with food administration in normal or obese subjects, but are negatively correlated with pituitary-adrenal activity. Clin Endocrinol 1997; 46:751-7.

28 Imbeault P. Environmental influences on adiponectin levels in humans. Appl Physiol Nutr Metab 2007; 32:505-11.

29 Guler N, Kirerleri E, Ones U, Tamay Z, Salmayenli N, Darendeliler F. Leptin: does it have any role in childhood asthma? J Allergy Clin Immunol 2004; 114:254-9.

30 Shore S, Schwartzman IN, Mellema M, Flynt L, Imrich A, Johnston R. Effect of leptin on allergic responses in mice. $J$ Allergy Clin Immunol 2005; 115:103-9.

31 Boulet LP. Asthma and obesity. Clin Exp Allergy 2013; 43:8-21.

32 Fenger RV, Gonzalez-Quintela A, Vidal $\mathrm{C}$ et al. Exploring the obesity-asthma link: do all types of adiposity increase the risk of asthma? Clin Exp Allergy 2012; 42:1237-45.

\section{Supporting Information}

Additional Supporting Information may be found in the online version of this article:

Data S1. Statistical methods.

Table S1. Within-area standardized Somers' $D$ of fatness measures with respect to adipokine exposures.

Figure S1. Somers' $D$ of exposures with respect to outcomes with adjustment type: Unadjusted.

Figure S2. Somers' $D$ of exposures with respect to outcomes with adjustment type: Propensity-adjusted (1).

Figure S3. Somers' $D$ of exposures with respect to outcomes with adjustment type: Propensity-adjusted (2).

Figure S4. Unadjusted Somers' $D$ of exposures with respect to Asthma in non-atopics and atopics.

Figure S5. Unadjusted Somers' $D$ of exposures with respect to ECRHS 5-point asthma score in non-atopics and atopics. 